\title{
HACIA LA CONSTRUCCIÓN DE UN CURRÍCULO PARA EL ÁREA DE GEOMETRÍA DE LA LICENCIATURA EN MATEMÁTICAS
}

\author{
Carmen Samper de Caicedo, Cecilia Leguizamón de Bernal, \\ Leonor Camargo, Alberto Donado* \\ Departamento de Matemáticas.
}

\begin{abstract}
Investigations in Mathematics Education have shown the necessity of joining, in a teachers training program, the disciplinary knowledge with the corresponding pedagogical reflection. Similarly they have shown the need for an epistemological approach to the disciplinary knowledge under the sociocultural and constructivist perspectives. This article presents the framework that underlies the proposal, in the field of Geometry, for the new Curricular Project of the undergraduate program in Mathematics Education of the Universidad Pedagógica Nacional, as well as the description of the courses that conform the proposal, in accordance with the aboye guidelines.
\end{abstract}

\section{RESUMEN}

Investigaciones en Educación Matemática han mostrado la necesidad de unir, en un programa de formación de profesores, el conocimiento disciplinar con la reflexión pedagógica correspondiente. Igualmente, han mostrado la necesidad de una aproximación epistemológica al conocimiento disciplinar bajo las perspectivas sociocultural y constructivista. Este artículo presenta el marco teórico que subyace la propuesta para la línea de Geometría, en el nuevo proyecto curricular de la Licenciatura en Matemáticas de la Universidad Pedagógica Nacional, así como la descripción de los cursos que la conforman, según las directrices mencionadas.

\section{INTRODUCCIÓN}

La posición de la geometría, frente a otras áreas en el currículo de las matemáticas, ha cambiado bastante durante las últimas cuatro décadas, en todos los niveles educativos. Con anterioridad a la década de los 60 , la geometría ocupaba un sitio preponderante en todos los programas de matemática en la secundaria y era parte esencial de la formación de los futuros profesores de matemáticas. En las siguientes dos décadas, con la introducción de la llamada Matemática Moderna, la geometría se fue diluyendo en el currículo escolar, apareciendo en forma fragmentada, a través de los cuatro primeros años de la Educación Básica Secundaria. En el panorama universitario, la geometría euclidiana fue abandonada en pro de teorías más generales y abstractas, como álgebra lineal, geometría diferencial, geometría algebraica y grupos de transformaciones. Fueron cursos enseñados, por topólogos o algebristas, muchas veces sin cimentar las bases en el estudio sistemático de los objetos geométricos específicos.

\footnotetext{
*Profesores Universidad Pedagógica Nacional.
} 
En particular, en la Universidad Pedagógica Nacional, el pénsum de geometría en el programa de formación inicial de profesores de matemáticas, ha sido objeto de varios cambios, reflejo de la preocupación constante por adecuar los programas a las inquietudes de la comunidad académica. En el programa vigente hasta el año 1986, cuyo énfasis se centraba en la formación matemática, se observa una fuerte influencia de la Teoría de Conjuntos y de la Matemática Moderna en el área de geometría. En los cursos de geometría se hacia un barrido de conceptos, partiendo de la geometría axiomática formal, pasando por la geometría vectorial en el plano y el espacio, el estudio del espacio vectorial 5)i' sobre li, sistemas axiomáticos para un espacio afín, para culminar, en Topología, con el estudio de espacios métricos y espacios métricos completos. (Universidad Pedagógica, 1977).

Con la reforma del año 86, influida por los desarrollos en el campo de la Educación Matemática hacia una recuperación de espacios propios para el estudio de la geometría euclidiana, se eliminaron de los cursos del área de geometría muchos de los temas que formaban parte del currículo de otras materias como Teoría de Conjuntos y Análisis. Se hicieron variantes en las asignaturas para enfatizar, en los primeros cursos, en la geometría euclidiana sintética además de la geometría analítica vectorial.

Actualmente los avances investigativos, en el campo de la Educación Matemática respecto de la preparación inicial del educador, han reconocido la necesidad de articular, en el programa de formación, el conocimiento disciplinar y la reflexión pedagógica, como vía indispensable para generar conocimiento profesional del futuro educador. De igual manera, han mostrado la necesidad de una aproximación epistemológica al conocimiento disciplinar de tipo socio-cultural y constructivista. Por esta razón, los profesores del Departamento de Matemáticas encargados del área de geometría, hemos asumido el reto de organizar, en el nuevo Proyecto Curricular de la Licenciatura, los espacios académicos de la geometría, para integrar en ellos una perspectiva amplia que aporte herramientas necesarias al futuro docente para su desempeño profesional en la educación básica y media.

Desde la misión que nos compete como entidad formadora de docentes, pretendemos contribuir con algunos lineamientos que consideramos indispensables en la formación inicial del futuro profesional de la educación matemática, con el objetivo de que éstos lleguen a influir en el panorama escolar. Teniendo en cuenta que ningún programa de geometría tiene validez universal, proponemos un currículo acorde con el tipo de estudiante que llega a nuestro programa de Licenciatura en Matemáticas, en el que se incluyen aspectos generales sobre los cuales hay actualmente un consenso amplio internacional. En este documento se recogen nuestros planteamientos iniciales, con la intención de generar un debate académico en la comunidad pedagógica interesada.

\section{PROPUESTA CURRICULAR PARA EL AREA DE GEOMETRÍA}

El Proyecto Curricular de la Licenciatura en Matemáticas de la Universidad Pedagógica Nacional se asume como un proyecto de investigación, que pretende buscar vías para la formación inicial del profesor de matemáticas orientadas, como lo propone Llinares (1998), a su actividad profesional. La búsqueda de una estructura para el área de geometría, en el programa de formación, está dirigida por el interrogante formulado por Niss (1998), según el cual el punto de partida para construir un curriculo ha de tener en cuenta respuestas a la pregunta: 
¿Cómo asegurar que los futuros pro fe-seres de matemáticas tengan una visten multifacética y bien fundamentada de la geometría, la habilidad para inventar una multitud de ambientes para la enseñanza y el aprendizaje de ésta, y la habilidad para evaluar la competencia geométrica de sus estudiantes? (Niss, 1998).

De este interrogante se desprenden otros más puntuales, que buscan determinar condiciones de enseñabilidad y educabilidad de la geometría en un programa de formación de docentes:

- ¿ Cómo puede hacerse la transposición didáctica de la disciplina, a dif e-rentes niveles escolares, sin distorsionarla?

- ¿ Qué características de los estudiantes pueden obstaculizar o favorecer la comprensión de la materia? (Farrell, 1987).

Para comenzar a dar respuesta a estos interrogantes se adopta, como se mencionó en el documento que describe el Proyecto Curricular de la Licenciatura en Matemáticas (Universidad Pedagógica, 1999), una organización sistémica, flexible y dinámica que, además de recoger los planteamientos de la Educación Matemática como referentes conceptuales e investigativos, adopte las directrices académicas de la Universidad Pedagógica Nacional. En este sentido, al proponer una estructura curricular para el área de geometría, se hace necesario identificar, como hipótesis inicial de trabajo, no sólo los diferentes espacios académicos que conducirían a la formación en el área, sino también algunos planteamientos y enfoques que posibiliten generar el ambiente propicio para lograr la formación deseada. A continuación se señalan algunos avances al respecto.

\section{Perspectiva del conocimiento geométrico}

La preparación académica de los futuros maestros debe proporcionarles una visión amplia de la geometría para que puedan responder a las exigencias del nivel en el cual se desempeñarán. Esto es posible si el programa brinda la oportunidad de experimentar actividades asociadas con los distintos dominios de la geometría y las relaciones entre ellos, para identificar las competencias geométricas que subyacen en su aprendizaje y poder hacer la transposición didáctica del saber geométrico en la escuela.

Un primer acercamiento a la caracterización de la geometría nos lleva a reconocer en ella varios dominios. La geometría puede ser vista como:

1) Una colección de características y propiedades de los objetos físicos, ya sean éstos naturales o creaciones del hombre. Esto hace a la geometría parte de la ciencia natural.

2) Un modelo de la realidad natural y artificial.

3) Una colección de diferentes teorías interrelacionadas con distintos fundamentos: geometría euclidiana y no euclidiana, y no euclidiana, geometría analítica, proyectiva, algebraica, diferencial, combinatoria.

4) Una componente constitutiva de la visión y visualización humana.

5) Un modelo prototipo de sistema axiomático deductivo.

6) Una herramienta de apoyo en las diferentes áreas de las matemáticas. 
Compartimos el punto de vista de Veblen (1934) quien integra los dominios antes mencionados en torno a dos campos estrechamente relacionados: el matemático y el de las ciencias naturales (figura 1). Esta relación puede evidenciarse a través de la evolución histórica de la geometría y de sus aplicaciones. En efecto, con los griegos, se dio el paso de la geometría de los objetos físicos a la geometría de los objetos geométricos y con el surgimiento de las geometrías no euclidianas, en el siglo XIX, se amplié la conceptualización de lo que es geometría, para dar paso a la geometría de las estructuras. (Moreno, sin fecha).

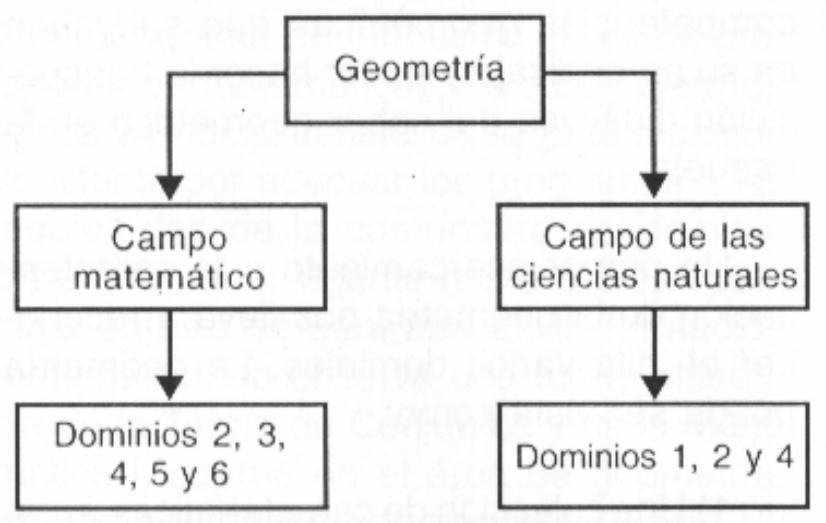

Figura 1.

Margaret Farrell (1987) amplia la apreciación anterior al considerar en la geometría dos aspectos inseparables:

i) El aspecto producto, como sistema formal ya construido, el cual se aplica a problemas teóricos y/o del mundo real.

ii) El aspecto proceso, el cual permite la extensión del conocimiento y la comprensión del mundo ideal de la matemática y del mundo real.

El esquema de la figura 2 (ver página siguiente) pretende ilustrar el mundo geométrico, bajo la visión entrelazada de estos autores. El diagramo clasifica los elementos que constituyen cada aspecto de la geometría. Dentro de la circunferencia se encuentran relacionados los elementos que forman un sistema deductivo. Es interesante ver que tales elementos se incluyen en el rectángulo inferior izquierdo, queriendo sugerir, de este modo, que pueden ser estudiados individualmente, mostrando la importancia que tienen por si mismos y no sólo como parte de una cadena deductiva. El incluir al mundo físico como parte del diagrama refleja la conexión de éste con el mundo matemático. Parte esencial del modelo son los procesos asociados a la actividad geométrica, listados en la parte derecha del esquema, como: conjeturar, experimentar, inducir, hipotetizar, simbolizar y abstraer.

El tratamiento de un tópico particular de la geometría debe vincularse a los procesos asociados a la actividad geométrica de los cuales se desprende su conceptualización, ya sea desde su caracterización como objeto matemático, identificando sus invariantes, las situaciones problema que le dan sentido y las representaciones asociadas (Vergnaud, 1997), o como parte de una cadena axiomática deductiva. 


\section{Planteamientos acerca del aprendizaje de la geometría}

Uno de los principios identificados por la Educación Matemática, respecto al aprendizaje de la matemática y en particular de la geometría, afirma que las nociones, los conceptos matemáticos y sus interrelaciones, y el rango de validez de las afirmaciones matemáticas, están inmersos y fuertemente influidos por las experiencias personales, matemáticas o no, de quien aprende.

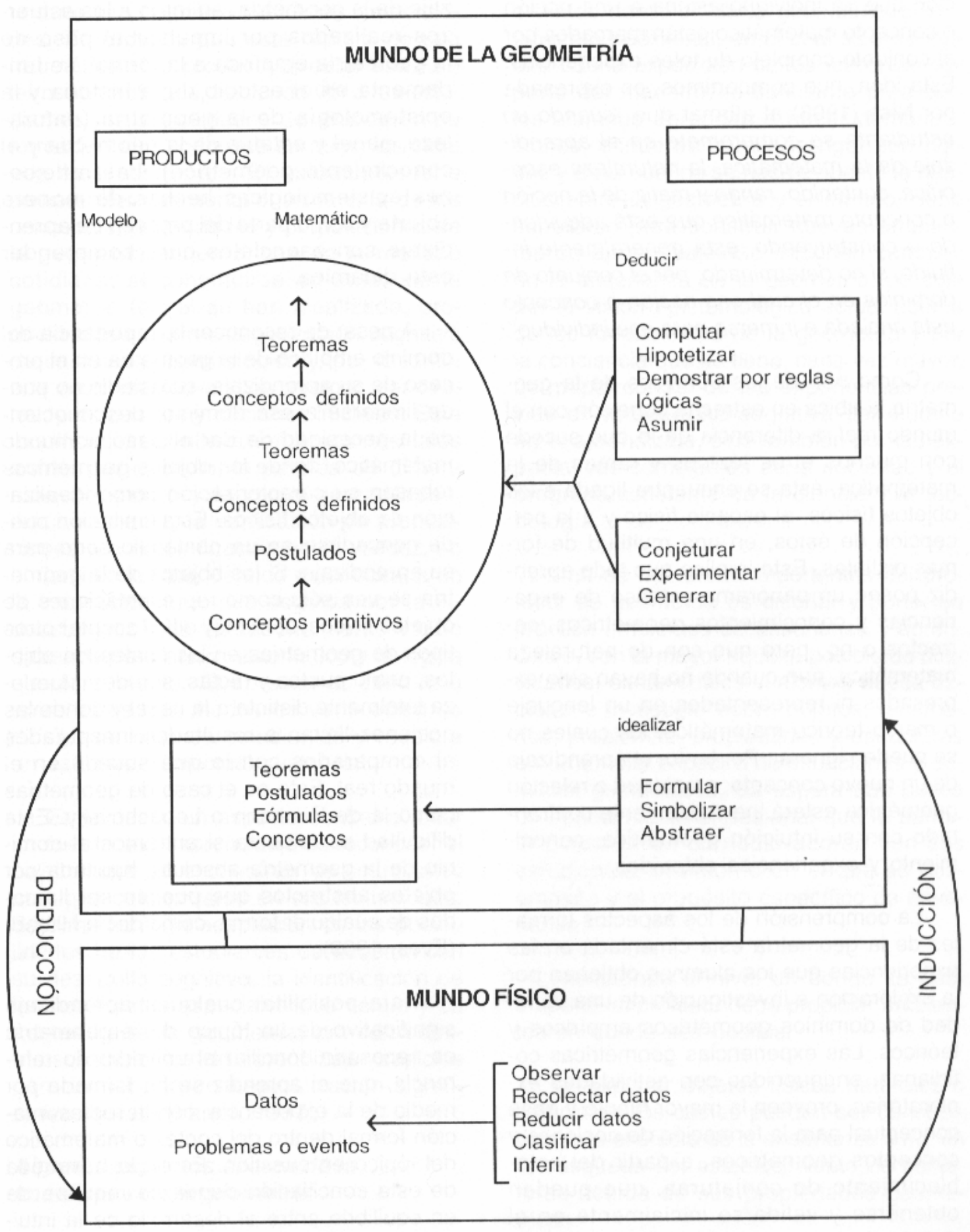

Figura 2. Modelo esquemático Farrell y Farmer. 
Por lo tanto, el significado y la interpretación que un individuo asigna a una noción o concepto matemático están marcados por el conjunto complejo de tales experiencias. Esta idea, que compartimos, es expresada por Niss (1998) al afirmar que cuando un estudiante se compromete en el aprendizaje de la matemática, la naturaleza específica, contenido, rango y matiz de la noción o concepto matemático que está adquiriendo o construyendo, está generalmente influido, si no determinado, por el conjunto de dominios en el cual esa noción o concepto está anclada e inmersa para ese individuo

Como uno de los dominios de la geometría la ubico en estrecha conexión con el mundo real, a diferencia de lo que sucede con muchos otros tópicos y ramas de la matemática, ésta se encuentra ligada a los objetos físicos, al espacio físico y a la percepción de éstos, en una multitud de formas distintas. Esto implica que todo aprendiz posee un panorama extenso de experiencias y conocimientos geométricos, correctos o no, pero que son de naturaleza matemática, aun cuando no hayan sido expresados ni representados en un lenguaje o marco teórico matemático, los cuales no se pueden ignorar. Por tanto, el aprendizaje de un nuevo concepto, propiedad o relación geométrica estará inevitablemente confrontado con su intuición geométrica, conocimiento y experiencias obtenidas.

La comprensión de los aspectos formales de la geometría está cimentada en las experiencias que los alumnos obtienen por la exploración e investigación de una variedad de dominios geométricos empíricos y teóricos. Las experiencias geométricas cotidianas, enriquecidas con actividades exploratorias, proveen la mayor fuente y base conceptual para la formación de nociones y conceptos geométricos, a partir del establecimiento de conjeturas, que pueden obtenerse y validarse inicialmente en el mundo de las experiencias del aprendiz y posteriormente en el marco de un sistema deductivo.

La dinámica del avance en el aprendizaje de la geometría, en torno a los esfuerzos realizados por impulsar el paso de la geometría empírica a la formal, se fundamento en el estudio de la historia y la epistemología de la geometría (naturaleza, papel y estatus de la geometría y el conocimiento geométrico). Las reflexiones epistemológicas hechas de manera abierta y como parte del proceso de aprendizaje son esenciales para comprender esta dinámica.

A pesar de reconocer ja importancia del dominio empírico de la geometría en el proceso de su aprendizaje, su estudio no puede limitarse a ese dominio, desconociendo la necesidad de dar el paso al mundo matemático, donde los objetos geométricos rebasan su caracterización como idealización de objetos físicos. Esta limitación puede convertirse en un obstáculo serio para su aprendizaje. Silos objetos de la geometría se ven sólo como representaciones de objetos físicos, será muy difícil aceptar otros tipos de geometrías en los cuales los objetos, como puntos y rectas, son de naturaleza totalmente distinta a la usual y donde las nociones llevan a resultados inesperados al compararlos con lo que sucede en el mundo real. Este es el caso de geometrías como la de Riemann o Lobachevsky. Esta dificultad se refuerza si entramos al dominio de la geometría absoluta, habitada por objetos abstractos que pueden ser llamados de cualquier forma, como decía Hilbert. (Eves, 1969)

Para posibilitar cualquier aprendizaje significativo de un tópico de la geometría es necesario conciliar el universo de referencia, que el aprendiz se ha formado por medio de la experiencia, con la representación formal dentro del contexto matemático del tópico en cuestión. Es en la búsqueda de esta conciliación donde se requiere de un equilibrio entre el desarrollo de la intuición bí y tridimensional y la investigación en la geometría formal, No 
puede desconocer-se que el paso de la dimensión manipulativa y visual a la teórica y formal implica una ruptura cognitiva de vastas proporciones. En orden a que esta ruptura no sea traumática sino productiva, debe estar totalmente motivada, haciendo ver a los estudiantes las posibilidades de avance en su formación al introducirse en dominios cada vez más abstractos. El estudiante debe comprender que aunque la mayoría de los conceptos, propiedades y proposiciones de la geometría tienen origen en la realidad cotidiana, al constituirse en entes de la geometría formal se han idealizado, produciéndose una recategorización cognitiva de carácter epistemológico y, por lo tanto, una modificación en los mismos conceptos, propiedades y nociones iniciales. Esta modificación conllevo implicaciones tales como, por ejemplo, la pérdida de dimensión del punto y la recta geométricas. Adicionalmente, la verificación de proposiciones y la determinación de su validez, en el dominio teórico, deja de estar basada en la inspección empírica seguida por la inducción. Lo que es razonamiento válido depende del sistema axiomático y la lógica que lo sustento. El apreciar la diferencia entre estos dos modos de verificación es difícil para muchos estudiantes y requiere un esfuerzo intelectual sustancial.

En síntesis, un aspecto central a tener en cuenta en el aprendizaje es reconocer el nivel en que se encuentran los estudiantes mas que concentrarse en sus jimitaciones. Sólo mediante la aceptación de las posibilidades de los estudiantes de acuerdo con su desarrollo cognitivo, la identificación de las habilidades espaciales que tienen y su actitud hacia la geometría, pueden construirse ambientes de aprendizaje propicios para iniciar una conceptualización significativa de ésta (Farrell, 1987)

\section{Algunos aspectos acerca de la enseñanza de la geometría}

En concordancia con las ideas contempladas para el aprendizaje de la geometría, su enseñanza debe propiciar ambientes que permitan a los estudiantes adquirir los conocimientos y habilidades propios de los distintos dominios de la geometría. El tratamiento tradicional, en el cual se cree que una buena exposición de los teoremas por parte del maestro es suficiente para que los alumnos aprendan a hacer demostraciones, donde se confunde rigor con rigidez y en el cual no se hace evidente la relación entre la geometría y el mundo, debe superarse para construir nuevos ambientes de aprendizaje. Se imponen cambios en la enseñanza de la geometría, no sólo por la visión epistemológica actual acerca de los fundamentos de la geometría y por la conciencia que se tiene, cada vez mayor, del papel central de las experiencias previas en la actividad cognitiva del alumno en el aprendizaje, sino también por las expectativas cambiantes de la sociedad y el progreso constante de la ciencia y la tecnología.

Una de las tareas importantes del profesor de geometría es diseñar y poner en práctica ambientes de enseñanza y aprendizaje, con la mayor riqueza posible de actividades, en las cuales el alumno pueda explorar e investigar sobre objetos, fenómenos, problemas, propiedades y estructuras en un amplio espectro de situaciones y dominios geométricos. Se espera que posea un conocimiento profesional que le permita construir un currículo acorde con sus estudiantes, el entorno en el que se desempeño y el propósito especifico de enseñanza.

Atendiendo al nivel en donde se desempeñe, el profesor debe propiciar ambientes en donde sea posible: 
- Explorar objetos físicos naturales o artificiales, los cuales pueden ser construidos para el efecto de la enseñanza o no, en la búsqueda del establecimiento de conjeturas acerca de sus propiedades geométricas.

- Utilizar herramientas tecnológicas que permitan visualizar y dar soporte a la experiencia geométrica.

- Construir situaciones que favorezcan el establecimiento de las relaciones entre la experiencia geométrica de los objetos físicos y la teoría geométrica formal.

- Establecer conexiones entre la geometría y otras ramas de la matemática para mostrar su importancia como apoyo en la construcción de relaciones aritméticas, algebraicas y analíticas además de la interpretación de fenómenos y/o resultados observados.

- Privilegiar, en la selección de teoremas a estudiar, aquellos que no sean intuitivos y que sean sorprendentes, para mostrar el poder del razonamiento sobre la experiencia física.

- Dar a los problemas matemáticos puros o aplicados diferentes tratamientos geométricos que permitan apreciar variedad de caminos para enfrentar la tarea.

- Descubrir que la visión humana y, por ende, muchas de las actividades del hombre están gobernadas por leyes y propiedades geométricas.

- Partir de modelos geométricos formales y estudiar hasta qué punto tiene sentido hacer una interpretación en la realidad física. Esto es, invertir el proceso mediante el cual se construyen los conceptos geométricos a partir de la experiencia.

- Favorecer el desarrollo de diversas formas de razonamiento tales como el razonamiento visual, el intuitivo e informal o el inferencial.

- Fomentar actividades enfocadas hacia la validación de las afirmaciones geométricas dentro de un sistema formal establecido.

- Examinar relaciones entre la teoría geométrica avanzada y la teoría geométrica elemental, con el fin de interpretar los conceptos y proposiciones de la geometría elemental en panoramas avanzados. Vale la pena insistir en la necesidad de establecer un puente entre la interpretación de las teorías geométricas avanzadas y las elementales y no dar por hecho que los estudiantes lo realizan por cuenta propia.

En síntesis, en la enseñanza de la geometría se han de aprovechar sus características especiales para el diseño de ambientes de aprendizaje. Cualquier situación geométrica provee oportunidades para hacer preguntas, inventar problemas, formular conjeturas, experimentar con casos particulares, argumentar y demostrar, entre otros. Dados su antigüedad y sus orígenes como ciencia empírica, la geometría está íntimamente ligada a la sociedad humana, a su historia, cultura, ciencia, tecnología, filosofía, arte y manualidades. Por tanto ofrece una amplia oportunidad para descubrir y relacionar las ideas matemáticas con la actividad humana y social. 


\section{Propuesta de espacios académicos}

El Proyecto Curricular de Licenciatura en Matemáticas de la Universidad Pedagógica Nacional contemplo dos ciclos de formación: uno de fundamentación y otro de profundización. En el primero se espera, entre otras cosas, "generar procesos que permitan revisar el conocimiento matemático que los estudiantes traen de sus experiencias académicas anteriores, con miras a la construcción de objetos matemáticos y a la integración de estos objetos con procesos matemáticos generales". El ciclo de profundización tiene entre sus objetivos afianzar la formación en matemáticas y en su didáctica, ahondando en una de las áreas del ambiente disciplinar.

A continuación se hace referencia a los diversos espacios académicos del Proyecte Curricular que tienen incidencia en la formación en Educación Matemática en lo que atañe a la geometría. Aún cuando se haga referencia a un espacio académico en particular, las ideas planteadas deben permear todo el ambiente del área en el Proyecto Curricular.

Proponemos que en el espacio académico Elementos de Geometría del ciclo de fundamentación, correspondiente al primer contacto de los estudiantes con la geometría en la universidad, se realicen procesos exploratorios para que los alumnos reconstruyan y amplíen el panorama geométrico, a través de temas variados dentro de un espectro amplio de los distintos dominios de la geometría. Como ya se mencioné, para que los alumnos comprendan la geometría formal, ésta debe estar arraigada con las experiencias que obtienen por la exploración e investigación de una variedad de dominios geométricos empíricos y teóricos, lo cual requiere de tiempo y de variados procedimientos metodológicos.

La experiencia docente con estudiantes que ingresan al programa nos ha mostrado que en su vida escolar anterior han tenido poca oportunidad de realizar actividades geométricas de tipo exploratorio. Por ello, cobran vital importancia las actividades en donde los alumnos, corten, peguen, plieguen, construyan y utilicen software especializado, pues éstas proveen la mayor fuente conceptual para la formación de las nociones, conceptos, proposiciones, métodos y técnicas geométricas, y para la posterior formalización de éstas, ya que dan la oportunidad de conjeturar sobre aquello que posteriormente será objeto de estudio. El aprendizaje significativo requiere de esfuerzos para que el alumno logre reconciliar la geometría empíricas y la teórica. No se pueden obviar etapas intermedias que permitan pasar gradualmente de los aspectos concretos e intuitivos a los aspectos abstractos y deductivos. Sólo puede obtenerse una comprensión global de los principales hechos, conceptos, procesos y estrategias geométricas, retomando los mismos tópicos desde varios enfoques y en varias etapas, en un proceso que podría parecer en espiral.

El contenido de Elementos de Geometría ha de ser flexible. Las temáticas pueden ser escogidas dentro de un abanico de posibilidades que abarquen temas como: congruencias, semejanzas, medida (geometría euclidiana), homotecias, simetrías, isometrías (geometría de las transformaciones), frontera, interior, exterior, distancia, invariantes (topología) geometrías finitas y geometrías no euclidianas, resolución de triángulos (trigonometría), lugares geométricos, cónicas (geometría analítica). Un tópico podrá ser cambiado por otro silos actividades de este último permiten desarrollar, en mejor forma, las metas propuestas.

Los conceptos, definiciones y teoremas trabajados en este espacio académico no necesariamente han de ser considerados como parte de un sistema formal, en el cual 
cumplen un papel; pueden ser tomados en forma independiente como herramientas para la resolución de problemas. Sin embargo, no se descarto la posibilidad de incluir la construcción de minisistemas axiomáticos; esto es, la utilización de deducciones locales en donde cada demostración depende de algunos presupuestos que han sido explícitamente dados como válidos. El estudio inicial de conceptos, definiciones y teoremas, sin que sean parte de un sistema deductivo, y de las relaciones de éstos con el mundo físico, a través de las aplicaciones, conducen al desarrollo de las competencias propias de quien hace geometría, para acceder, entre otros propósitos, a la construcción de un sistema formal.

Como se espera que a lo largo de la carrera los estudiantes alcancen niveles de razonamiento que les permita hipotetizar, razonar deductivamente y comprender el rol de los modelos matemáticos, este primer espacio académico da la oportunidad de experimentar acercamientos informales que proporcionen los conocimientos y habilidades de razonamiento, cercanos al razonamiento formal, los cuales son indispensables para abordar los siguientes espacios académicos.

Para establecer el vínculo entre Elementos de Geometría y espacios académicos en donde se hará uso de otros modelos geométricos desligados de la intuición del mundo cercano, los cuales darán lugar al estudio de las estructuras geométricas abstractas, se proponen los siguientes dos espacios académicos, Geometría Plana y Geometría del Espacio, en los cuales la Geometría de Euclides se constituye en la base para la construcción de un sistema deductivo. Esta se selecciono porque los elementos con los que trabaja tienen un soporte intuitivo y es posible la visualización de situaciones geométricas en el mundo físico, las cuales pueden ser representadas por medio de dibujos, constituyéndose en modelos concretos materializables". Un propósito de estos espacios académicos es el uso de la Geometría de Euclides como herramienta para la adquisición de elementos necesarios para acceder a otros modelos, algunos de índole algebraico, referidos a la geometría euclidiana o no euclidiana (Riemann, Lobachevsky) o a otros sistemas deductivos propios de cualquier teoría matemática.

Los espacios académicos Elementos de Geometría, Geometría Plana y Geometría del Espacio son fuente para establecer relaciones entre las diversas geometrías euclidianas, las cuales surgen en el contexto educativo al hacer la transposición didáctica, como versiones que tienen, tanto profesores como autores de textos, respecto a qué de la Geometría de Euclides puede enseñarse en la escuela. Difieren de la de Euclides en cuanto se amplía el conjunto de postulados, incluyendo en éste afirmaciones que en la geometría de Euclides son teoremas. De esta manera se simplifico el sistema deductivo que se pretende desarrollar con los alumnos. No se puede olvidar que estamos formando futuros profesores y las actividades que los estudian-tos experimenten y desarrollen se convierten en semillero de ideas para el diseño de ambientes de aprendizaje en el desempeño de su labor profesional. Las habilidades básicas visuales, verbales, gráficas, lógicas y de aplicación i.J uiridas enriquecerán su futura labor.

El siguiente espacio académico, Geometría Analítica, con el cual culmino el Ciclo de Fundamentación, permite abrir el campo para el estudio de espacios $n$-dimensionales. En ella se pretende enfatizar en el paso de contextos geométricos a contextos algebraicos mediante el uso de coordenadas y de las herramientas del cálculo vectorial, para resolver problemas geométricos. Se intenta explorar y utilizar las ventajas que aporta la mirada cartesiana de la geometría, sus objetos e irterrelaciones. 
Como todas las actividades propuestas en el Proyecto Curricular están encaminadas a la formación integral de un profesional de la Educación Matemática, los espacios académicos descritos y aquellos diseñados para la reflexión pedagógica han de proporcionar bases para el análisis de la enseñanza y el aprendizaje de la geometría en la Educación Básica y Media. En el espacio académico Matemáticas Escolares se estudiarán, junto con temas de las demás áreas del currículo escolar, aquellos elementos que posibilitan la adaptación de temas geométricos a cada nivel, sin perder de vista las características de la disciplina. Se reflexionará sobre el medio escolar y el desarrollo de las funciones cognitívas propias de la actividad geométrica. Se espera consolidar los elementos que ayuden a diseñar ambientes adecuados para introducir nuevos conceptos, teniendo en cuenta los conocimientos previos de los alumnos, las diferentes formas que tienen para acceder a ellos, con el objeto de establecer comunicación eficaz y captar el interés.

El espacio académico, Componente de Integración, tiene por objetivo que los alumnos entiendan y aprovechen las interrelaciones entre las distintas áreas de la matemática. El aporte de la interpretación geométrica en la visualización inmediata de operaciones, procesos, reglas, fenómenos y resultados, en otras áreas de la matemático es innegable.

El currículo del plan de formación debe incentivar en espacios como, Epistemología. la reflexión sobre la historia y la epistemología de la geometría. Se recomienda abordar temas como: orígenes empíricos de la geometría, fundamentos geométricos de la matemática griega, desarrollo y significado de la geometría sintética euclidiana, desarrollo y significado de geometrías no-euclidianas, papel de la geometría en otras ramas de la matemática y viceversa.

Los tres espacios académicos del ciclo de profundización del área de geometría, Electivas, están diseñados para profundizar en tópicos de geometría vectorial, geometrías no euclidianas, topología, entre otros. Ellos permitirán a los estudiantes conocer aspectos específicos acerca de diferentes teorías geométricas y sus interrelaciones. La transferencia entre diferentes clases de códigos y modelos geométricos como los de lo geometría vectorial, la geometría proyectiva o algebraica, serán de gran utilidad a la hora de resolver problemas matemáticos puros o aplicados desde diferentes tratamientos geométricos. En topología se aprovecha el trabajo desarrollado previamente en el ciclo de fundamentación en las áreas de geometría y cálculo, para trabajar con las abstracciones de conceptos clásicos del estudio de funciones reales y complejas (abiertos, continuidad, espacios métricos) y estudiar las propiedades que se preservan bajo diversas transformaciones (homomorfismos).

Finalmente, al igual que para todas las áreas del ambiente disciplinar, la de geometría culmino con el espacio académico Didáctica de la Geometría, en donde se reflexiona sobre las interrelaciones docente, alumno y conocimiento geométrico, tomando como referencia las experiencias vividas por los estudiantes en sus prácticas pedagógicas y en el programa de formación y los desarrollos del campo investigativo de la didáctica de la geometría.

\section{Algunos elementos para la evaluación de los estudiantes en el curso Elementos de Geometría}

El cambio de paradigma, al pensar en un currículo en términos del aprendizaje y no únicamente en términos de la enseñanza, implica un cambio radical del concepto de evaluación, pues obliga a tener en cuenta la actividad cognitiva del estudiante. Este hecho 
pone de presente la gran complejidad de la tarea evaluativa y la dificultad para establecer claramente los aspectos a considerar.

Por mucho tiempo, los criterios que se emplearon para evaluar a los alumnos se confundieron con los criterios de validación que se emplean en la investigación matemática. Por ejemplo, para medir el aprendizaje de un estudiante en geometría se realizaban pruebas escritas en donde se observaba qué tanto se acercaba su producción a la presentación formal que se usa para comunicar los resultados del trabajo geométrico. La organización lógica establecida por la comunidad de matemáticos para validar sus resultados pasó a ser criterio de validación del aprendizaje de los estudiantes. No se tuvo en cuento que hacer ese tipo de demostraciones, con el consiguiente nivel de rigor, es una actividad inútil si no hay una previa construcción de significados matemáticos, proceso que conllevo una lenta evolución conceptual de las ideas matemáticas.

Con el ánimo de plantear un debate académico y conscientes de que es una primera aproximación al tema, el grupo de geometría propone considerar los siguientes aspectos para evaluar a los estudiantes:

Comunicación: Resaltamos este aspecto porque reconocemos que la matemática es una actividad social que evoluciono como fruto de la expresión de ideas y consensos logrados, con el fin de construir significados compartidos. En este aspecto queremos valorar:

- la evolución de la capacidad de comunicar a otros en forma oral y escrita las ideas geométricas,

— el uso del lenguaje matemático acordado para el trabajo geométrico,

- la incorporación en el lenguaje de los términos asociados a los objetos, propiedades o relaciones geométricas con el objeto de buscar precisión en la comunicación,

— el uso, con significado, de conceptos, propiedades y relaciones geométricas.

La evaluación de todo lo anterior refleja, en cierto sentido, el grado de dominio de un concepto, propiedad o relación, porque la claridad en la expresión de las ideas a otros es un indicativo de la conceptualización realizada.

Representación: Otro indicador de la conceptualización de los objetos, propiedades y relaciones geométricas es el tratamiento de las diversas representaciones asociadas, en tanto que sólo es posible acceder a éstos por medio de ellas (Duvol, 1999). El significado que tenemos de un objeto, propiedad o relación geométrica es una síntesis de la información que se extrae de las diferentes representaciones. Por todo lo anterior, es fundamental valorar en la actividad representativa:

- la información acerca de un objeto, propiedad o relación geométrica que se extrae de la representación verbal del mismo (definición),

— la información que se extrae de una figura geométrica,

- el progreso en la habilidad para manipular, interpretar semántica y sintácticamente las representaciones matemáticas, 
- la evolución conceptual en términos de reconocer las figuras geométricas como representaciones de entes geométricos y no como dibujos de objetos concretos.

La evaluación de estos aspectos nos permitirá evidenciar la capacidad de traducción y transformación de un sistema de representación a otro.

Resolución de Problemas: Aunque la resolución de problemas puede considerar-se como una metodología de trabajo, también puede verse como una actividad cognitiva, propia del trabajo matemático. Este es el aspecto en el que queremos valorar cómo evoluciona el estudiante en cuanto a:

- la comprensión del enunciado de los problemas en términos de distinguir, en el caso de las demostraciones, la hipótesis de la tesis, y, en el caso de los problemas de aplicación, el conocimiento geométrico subyacente,

- la identificación del conocimiento geométrico previo que está relacionado con el enunciado del problema,

— las estrategias de resolución,

— la incorporación de argumentos y conocimiento geométricos en las explicaciones,

— la habilidad para inventar y formular problemas.

La evaluación de estos aspectos nos permitirá evidenciar la evolución en el aprendizaje del oficio del matemático: resolver problemas.

Razonamiento: La geometría es un campo fructífero para desarrollar las habilidades de razonamiento, tanto de las formas argumentativas básicas poro explicar y convencer a otros en el marco del discurso cotidiano, como de las estrategias para demostrar una afirmación, en el marco propiamente geométrico, en donde se privilegio la prueba deductiva. Se quiere ver la evolución en los siguientes aspectos:

- las estrategias de razonamiento visual,

- el establecimiento de conjeturas,

- la argumentación de tipo informal con el uso de instrumentos de mediación para verificar o explicar una conjeturo,

- las argumentaciones de tipo deductivo,

— el interés y la motivación por hacer demostraciones

La evaluación de estos aspectos es un indicador del reconocimiento, por parte de los estudiantes, de la importancia y la necesidad de utilizar la demostración formal como la forma válida de comprobar enunciados geométricos.

Actitud pedagógica: Se espera observar si el estudiante evoluciono en su compromiso por el aprendizaje y la enseñanza de la geometría pues la educación matemático será su campo de actuación. Formar un maestro reflexivo e indagador implica ir construyendo una actitud inquisitiva en aspectos como: 
— el análisis crítico de los discursos geométricos de los textos escolares,

- el análisis y evaluación de las formas de razonar y de las estrategias de resolución de problemas, de otras personas.

- las formas para lograr la transposición didáctica de temas geométricos,

- las problemáticas asociadas al aprendizaje y la enseñanza de temas geométricos.

El avance en estos indicadores se refleja no sólo en la forma como los alumnos asumen las tareas respectivas a este aspecto sino también en su actitud de compartir conocimiento y el esfuerzo por hacer comprensibles las explicaciones a sus pares.

Cabe resaltar que a través de la valoración de los aspectos considerados anteriormente se está evaluando, entre otras cosas:

- el aprendizaje de nociones, conceptos, definiciones, métodos y teoremas característicos de los diferentes tópicos de la geometría,

- la comprensión de los fundamentos y nexos entre los diferentes tópicos,

- la visión de las interrelaciones de la geometría con otras ramas de la matemática,

- la comprensión de la naturaleza dual de la geometría como punto de encuentro entre la matemática teórica y abstracta, y la modelación del espacio físico, los objetos y fenómenos en la naturaleza,

- la apreciación de la matemática como disciplina, su naturaleza y su papel en la sociedad,

- la actitud hacia la matemática,

- el desarrollo de la autoestima y la autonomía.

\section{CONCLUSIONES}

Las ideas esbozadas en este artículo muestran un cambio en la forma de concebir una propuesta curricular paro el área de geometría en un programa de formación de maestros. De organizaciones curriculares centradas en contenidos, organizados en una secuencio lógica de la disciplina, se da paso o una propuesta enfocada hacia el aprendizaje, la cual atiende a la caracterización de los estudiantes que ingresan al programo y al sentido mismo del proyecto curricular: formar maestros. Por esta última razón, la reflexión sobre el aprendizaje y la enseñanza de la geometría está presente desde el primer momento de la formación.

Para cumplir con las metas establecidas para el programo es necesario generar un ambiente universitario que permita al estudiante formarse seguro de si mismo, independiente, entusiasta, reflexivo, critico y poseedor de herramientas conceptuales, aspectos que le permitirán gestionar, en un futuro, un currículo acorde con las condiciones de sus educandos. Este perfil se logro silo forma de trabajo estimula la disposición al aprendizaje continuo, la responsabilidad individual frente a los resultados de su aprendizaje y el trabajo en equipo, considerado como el espacio que favorece lo discusión, la búsqueda de consensos, el respeto por las ideas de los demás y la reflexión colectiva. Este estilo de formación contribuirá al desarrollo de la capacidad prepositiva para innovar las prácticas de enseñanza usuales y lograr cambios acordes con las necesidades del medio, generar actividades de enseñanza y de aprendizaje y diseñar matemáticas escolares. La auto-confianza, la capacidad de reflexión, el espirito indagador 
y el conocimiento significativo de la geometría son prerrequisitos para actuar con autonomía en los procesos de transformación de la realidad.

\section{REFERENCIAS BIBLIOGRÁFICAS}

- Bartolini, M Boero, P. (1998). Teaching and Learning Geometry in Context. En MAMMANA C; VILLANI V (eds). Perspectives on the Teaching of Geometry for the $21^{\text {st }}$ Century. Kluwer Academic Publishers, Netherlands.

- Bishop, A. (1986). ¿Cuáles son algunos de los obstáculos para el aprendizaje de geometría?'. En: Morris, R. (ed). Estudios en Educación Matemática: Enseñanza de la geometría. UNESCO.

- Duval, R. (1999). Semiosis y Pensamiento Humano. Registros semióticos y aprendizajes intelectuales. Universidad del Valle; Instituto de Educación y Pedagogía.

- Eves, H. (1969) Estudio de las Geometrías. México: Uteha.

- Farrell, M. (1987). Geometry for Secondary School Teachers". En: Lindquist, M.; Shulte, A. (eds). Learning and Teaching Geometry, K-12. 1987 Yearbook, NCTM, Virginia.

- Llinares, S. (1998). Conocimiento profesional del profesor de matemáticas y procesos de formación". En: Revista de Didáctica de las Matemáticas, No. 17.

- Meserve, et al. (1986). La formación del docente y la enseñanza de la Geometría". En: Morris, R. (ed). Estudios en Educación Matemática, Enseñanza de la Geometría, UNESCO, vol. 5.

- Moreno, L. (sin fecha). Una perspectiva sobre la demostración. México: CINVESTAV.

- Niss, N. (1998). "Teacher Qualifications and the Education of Teachers". En: Mammana, C. et al. Perspectives on the Teaching of Geometry for the $21^{\text {st }}$ Century. Kluwer Academic Publishers, Netherlands.

- Niss, M. (1998). Dimensions of Geometry and Assesments". En: Mammana, C. et al. Perspectives en the Teaching of Geometry for the $21^{\text {st }}$ Century. Kluwer Academic Publishers, Netherlands.

- Veblen, O. (1934). The modern approach to elementary geometry". En: Lindquist, M.; Shulte, A. (eds). Learning and Teaching Geometry, K-12. 1987 Yearbook, NCTM, Virginia.

- UNIVERSIDAD PEDAGÓGICA NACIONAL (1977). Información Académica. Departamento de Matemáticas. UPN.

- UNIVERSIDAD PEDAGÓGICA NACIONAL (1999). Licenciatura en Matemáticas. Proyecto Currícular. Departamento de Matemáticas. Facultad de Ciencia y Tecnología. 
- Vergnaud, G. (1997). The Nature of Mathematical Concepts". En: Nunes, T. et al. (1997) Learning and Teaching Mathematics: An International Perspective, Psychology Press. 